\title{
Secreted calmodulin-like skin protein inhibits neuronal death in cell-based Alzheimer's disease models via the heterotrimeric Humanin receptor
}

\author{
Y Hashimoto ${ }^{1}$, M Nawa ${ }^{1}$, M Kurita $^{1}$, M Tokizawa ${ }^{1}$, A Iwamatsu ${ }^{2}$ and M Matsuoka ${ }^{\star, 1}$
}

Humanin is a secreted bioactive peptide that is protective in a variety of death models, including cell-based neuronal death models related to Alzheimer's disease (AD). To mediate the protective effect in AD-related death models, Humanin signals via a cell-surface receptor that is generally composed of three subunits: ciliary neurotrophic factor receptor $\alpha$, WSX-1 and gp130 (heterotrimeric Humanin receptor; htHNR). However, the protective effect of Humanin via the htHNR is weak $\left(\mathrm{EC}_{50}=1-10 \mu \mathrm{m}\right)$; therefore, it is possible that another physiological agonist for this receptor exists in vivo. In the current study, calmodulin-like skin protein (CLSP), a calmodulin relative with an undefined function, was shown to be secreted and inhibit neuronal death via the htHNR with an $\mathrm{EC}_{50}$ of 10-100 pм. CLSP was highly expressed in the skin, and the concentration in circulating normal human blood was $\sim 5 \mathrm{~nm}$. When administered intraperitoneally in mice, recombinant CLSP was transported across the blood-cerebrospinal fluid (CSF)-barrier and its concentration in the CSF reaches $1 / 100$ of its serum concentration at $1 \mathrm{~h}$ after injection. These findings suggest that CLSP is a physiological htHNR agonist.

Cell Death and Disease (2013) 4, e555; doi:10.1038/cddis.2013.80; published online 21 March 2013

Subject Category: Neuroscience

Humanin, a 24-amino acid peptide, was originally cloned as a secreted factor that inhibited death of cultured neuronal cells, induced by the expression of a London-type familial Alzheimer's disease (AD)-linked mutant gene of the amyloid $\beta(A \beta)$ precursor protein (APP), V642I-APP. ${ }^{1,2}$ Since then, a number of studies have shown that Humanin is protective in a variety of cell-based death models, including non-neuronal death models $^{3-7}$ and AD-related neuronal death models (for review see Nishimoto et al. $^{8}$ and Matsuoka ${ }^{9,10}$ ). Furthermore, Humanin inhibits cell death in various in vivo models, including a mouse stroke model, ${ }^{11,12}$ a rodent model of testicular germ cell death caused by exposure to a gonadotropin-releasing hormone antagonist $^{13}$ and a mouse model of ischemic heart disease. ${ }^{14}$

Humanin has other functions aside from inhibition of cell death. It regulates the peripheral insulin signaling by acting through the central nervous system $(\mathrm{CNS})^{15}$ and preserves endothelial function and prevents atherosclerotic plaque progression in hypercholesterolemic apolipoprotein E-deficient mice. ${ }^{16}$ Humanin ameliorates the mouse memory impairment, caused by an injected muscarinic receptor antagonist ${ }^{17-19}$ and intracerebroventricularly administered $\mathrm{A} \beta,{ }^{20-23}$ and it also ameliorates the memory impairment in familial AD transgenic mice. ${ }^{24-26}$ Memory impairment in the muscarinic receptor antagonist-induced dementia mouse model, which is caused by the failure of cholinergic neurotransmission. Memory impairment in the AD transgenic-modeled mice is also mainly caused by the synaptic dysfunction-mediated impairment of cholinergic neurotransmission in the CNS. ${ }^{27}$ Therefore, it is highly likely that Humanin also rescues cholinergic dysfunction in these dementia models. ${ }^{24}$

Humanin mediates its protective effect by binding to its specific receptor or target. Three putative Humanin receptors have been reported. Cytosolic Humanin partly inhibits staurosporine-induced cell death by binding to Bax and neutralizing the Bax-mediated proapoptotic pathway. ${ }^{28}$ Secreted Humanin binds to cell-surface formyl peptide receptor-like- 1 and inhibits $\mathrm{A} \beta$-induced death of $\mathrm{PC} 12$ neuronal cells. ${ }^{29}$ The neuroprotective effect of Humanin against $A D$-related neuronal death occurs via a cell-surface receptor that belongs to the interleukin (IL)-6 receptor family. ${ }^{30,31}$ This receptor, called as the heterotrimeric Humanin receptor (htHNR), is comprised of ciliary neurotrophic factor receptor $\alpha$ (CNTFR), WSX-1 and gp130, all of which are expressed in the neurons. The activity and expression of Humanin can be affected by the binding of other proteins to Humanin; for example, insulin-like growth factor-binding protein-3 functionally interacts with Humanin, ${ }^{32}$ tripartite motif-containing protein 11 destabilizes Humanin protein, ${ }^{33}$ and $\mathrm{V}$-set and transmembrane domain containing 2 like protein (VSTM2L) inhibits Humanin activity. ${ }^{34}$

There have been some concerns about whether endogenous levels of Humanin are neuroprotective in AD.

\footnotetext{
${ }^{1}$ Department of Pharmacology, Tokyo Medical University, Shinjuku-ku, Tokyo, Japan and 2Protein Research Network, Midori-ku, Yokohama, Kanagawa, Japan ${ }^{*}$ Corresponding author: M Matsuoka, Department of Pharmacology, Tokyo Medical University, 6-1-1 Shinjuku, Shinjuku-ku, Tokyo 160-8402, Japan. Tel: + 81-3-3351-6141; Fax: + 81-3-3352-0316; E-mail: sakimatu@ tokyo-med.ac.jp

Keywords: Humanin; calmodulin-like skin protein (CLSP); neuronal death; Alzheimer's disease

Abbreviations: AD, Alzheimer's disease; A $\beta$, amyloid beta; APP, amyloid beta precursor protein; CNS, central nervous system; CSF, cerebrospinal fluid; CNTFR, ciliary neurotrophic factor receptor $\alpha$; CLSP, calmodulin-like skin protein; htHNR, heterotrimeric Humanin receptor; HNG, Humanin G; hCLSP, human calmodulin-like skin protein; i.n., intranasal; i.p., intraperitoneally; mCLSP, mouse calmodulin-like skin protein

Received 17.9.12; revised 10.2.13; accepted 18.2.13; Edited by A Verkhratsky.
} 
Humanin is only weakly neuroprotective against models of $A D$ with a half maximal effective concentration $\left(E C_{50}\right)$ of $1-$ $10 \mu \mathrm{m}^{1,2}$ In addition, Humanin is weakly expressed in the CNS, ${ }^{35}$ whereas it is strongly expressed in the testis. ${ }^{13,35,36}$ These findings suggest that the concentration of Humanin might be insufficient to confer a protective effect in the CNS.

In the current study, we show that calmodulin-like skin protein (CLSP), which is secreted by skin keratinocytes and circulated in the blood, displays the htHNR agonist activity with an $\mathrm{EC}_{50}$ of 10-100 pm against $A D$-related neuronal death, which indicates that CLSP is $10^{4}-10^{5}$ times more potent than Humanin. Furthermore, intraperitoneally (i.p.) injected CLSP is transported across the blood-cerebrospinal fluid (CSF) barrier into the CNS. These data support the idea that CLSP may serve as a physiological agonist for the htHNR in the CNS.

\section{Results}

Human CLSP is immunoprecipitated with a Humanin antibody. Previously, several proteins were recognized by immunoblot analysis with a Humanin polyclonal antibody PO4 (raised against the entire Humanin peptide). ${ }^{35}$ Herein, we attempted to identify Humanin-like proteins in various cells and tissues by performing mass spectrometry analysis of the PO4 immunoprecipitates. Under the employed conditions, PO4 did not immunoprecipitate Humanin (3 kDa) from human cells or the Humanin homolog from mouse tissues. However, PO4 immunoprecipitated a $12 \mathrm{kDa}$ protein and a $8 \mathrm{kDa}$ protein from human SF268 glioblastoma cells and human teratocarcinoma NT2 cells, respectively (Figure 1a). Both the proteins were identified as fragments of human CLSP (hCLSP), which is a calmodulin-like protein containing EF-hand $\mathrm{Ca}^{2+}$-binding motifs and is mainly expressed in skin keratinocytes ${ }^{37,38}$ (Figure 1b; Supplementary Figures S1-3 for detailed data of mass spectrometry analysis).

As hCLSP was cross reactively recognized by the Humanin antibody in the immunoprecipitation (Figure 1), we next examined whether similarities in amino acid sequence were present between Humanin and hCLSP. Amino acids 3-19 of Humanin (MAPRGFSCLLLLTSEIDLPDVKRRA) is the core region of Humanin, necessary and sufficient for its activity. ${ }^{2,8}$ hCLSP, a 146-amino acid protein, contains a domain (NLSEAGLRKLISEVDGD) between 43-59 amino acids (Figure $1 \mathrm{~b}$ ), which is minimally homologous to the 17-amino acid core region of Humanin (Figure 1c). Mutation of the Pro3, Ser7, Cys8, Leu9, Leu12, Thr13, Ser14 or Pro19 of Humanin to alanine nullifies the Humanin activity. ${ }^{2,8}$ Among these important amino acids, Leu9, Leu12 and Ser14 are conserved in hCLSP (Figure 1c).

V642I-APP-induced neuronal death is inhibited by coexpression of hCLSP. Consistent with our previous study, ${ }^{30,31}$ the expression of V642I-APP-induced death of human SH-SY5Y neuroblastoma cells as assessed by counting viable cells that were attached to culture plates (Figure $2 \mathrm{a}$ ) and by the WST-8 cell viability assay (Figure $2 \mathrm{~b}$ ). Coexpression of hCLSP with V642I-APP, inhibited V642I-APP-induced death of SH-SY5Y cells (Figures 2a a

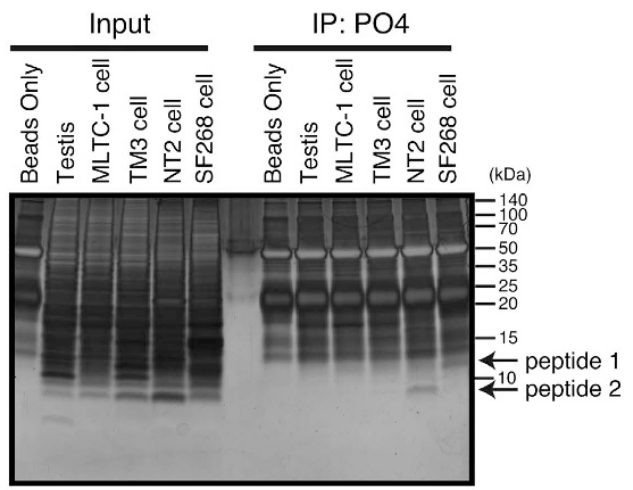

b

1 MAGELTPEEEAQYKKAFSAV 20

21 DTDGNGTINAQELGAALKAT 40

41 GKNLSEAQLRKLISEVDGDG 60

61 DGEISFQEFLTAARKARAGL 80

81 EDLQVAFRAFDQDGDGHITV 100

101 DELRRAMAGLGQPLPQEELD 120

121 AMIREADVDQDGRVNYEEFA 140

141 RMLAQE

146

C

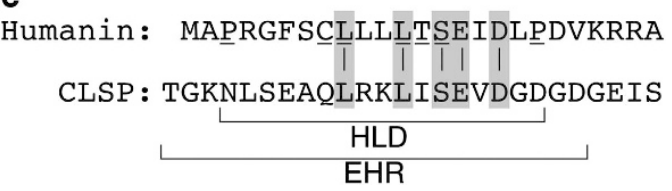

Figure 1 A Humanin antibody immunoprecipitates human CLSP. (a) Cell lysates from mouse testis and cultured cells were subjected to the immunoprecipitatation with the Humanin antibody PO4. The resulting immunoprecipitates and input lysates were analyzed using SDS-PAGE and silver staining. Mass spectrometric analysis revealed that two proteins (named peptide 1 and 2; arrows) were fragments of human CLSP (hCLSP) (Supplementary Figures S1-3). (b) The primary amino acid sequence of a hCLSP isoform. The underlined region is minimally similar to the core region of Humanin. Two single-nucleotide polymorphisms in the hCLSP gene exist, in which the $58^{\text {th }}$ glycine and the $74^{\text {th }}$ arginine are replaced by serine and lysine, respectively, in certain isoforms. (c) The comparison of Humanin and the Humanin-like domain (HLD) of hCLSP. Mutation of an underlined amino acid of Humanin to alanine nullifies Humanin activity. ${ }^{2}$ The amino acids that are identical between Humanin and hCLSP are marked with black squares. HLD: Humanin-like domain, EHR: endogenous Humanin-like region of hCLSP that appears in Figure $3 \mathrm{~b}$

and b). Immunoblot analysis excluded the possibility that the expression of hCLSP inhibited cell death by downregulating V642I-APP expression (Figure 2c).

hCLSP is secreted. Because Humanin is secreted and acts extracellularly, ${ }^{1,2,8,39}$ we investigated whether hCLSP was secreted. To this end, C-terminally FLAG-tagged hCLSP and Humanin were expressed in SH-SY5Y cells. Humanin-FLAG and CLSP-FLAG were immunoprecipitated from the conditioned medium (CM) by the FLAG-antibody-conjugated beads (M2 agarose) (Figure 3a), suggesting that hCLSP is secreted, similar to Humanin. The treatment with $(+)$-Brefeldin A (BFA), an inhibitor of endoplasmic reticulum-Golgi transport involving the conventional secretion pathway, ${ }^{40}$ reduced the secreted amounts of hCLSP and Humanin (Figure 3a). These results suggest that hCLSP and 
a

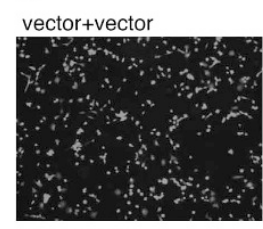

V642I-APP+vector

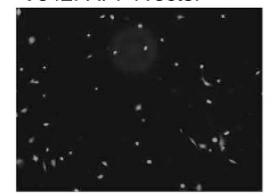

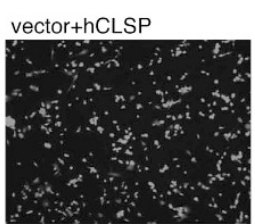

V642I-APP+hCLSP

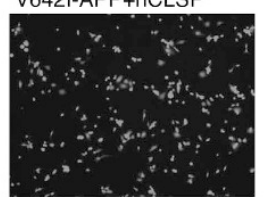

b

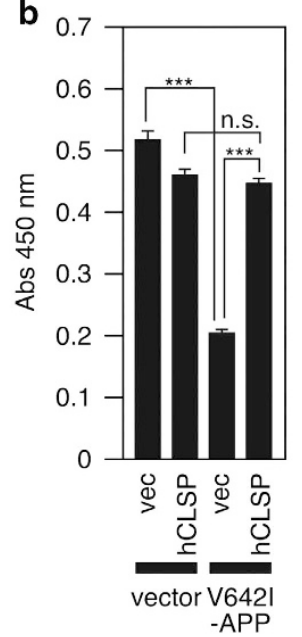

c
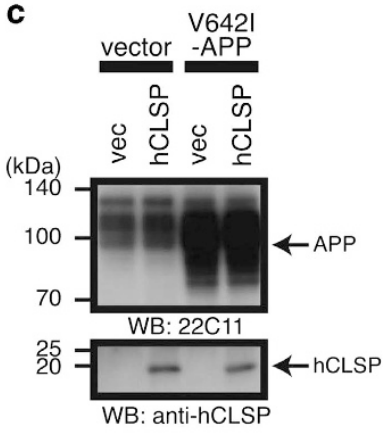

Figure 2 Coexpression of hCLSP with V642I-APP inhibits V642I-APP-induced neuronal death. (a-c) SH-SY5Y cells, co-transfected with the empty pcDNA3 vector (vector) or pcDNA3-V642I-APP (V642I-APP) together with the pcDNA3.1/MycHis vector (vector) or pcDNA3.1/MycHis-hCLSP (hCLSP), were harvested for microscopic analysis (a) and WST-8 assays (b) ${ }^{* * \star} P<0.001$; n.s., not significant. The cell lysates were subjected to SDS-PAGE and immunoblot analysis with an antibody to APP (antibody 22C11) or CLSP (c)

Humanin are secreted via the conventional secretion pathway, even though hCLSP, unlike Humanin, ${ }^{1,2,8,39}$ lacks a typical secretion signal sequence (Figure 1b).

We previously showed that Humanin tended to be detected as a dimer and occasionally a multimer even in the SDSPAGE gel. ${ }^{35,41}$ In the current study, we also found that hCLSP and Humanin-FLAG were detected as a dimer and a multimer in the Tris/Tricine-SDS-PAGE (Figure 3a).

Secretion of hCLSP is essential for hCLSP activity. We constructed vectors encoding deletion mutants of hCLSP (all C-terminally tagged with MycHis); namely, hCLSP- $\triangle \mathrm{C} 1$, hCLSP- $\Delta \mathrm{C} 2$ and $\mathrm{hCLSP}-\Delta \mathrm{N} 1$, in which $\mathrm{C}$-terminal regions (a.a. 79-146 and a.a. 62-146) and an N-terminal region (a.a. 2-39) were deleted, respectively (Figure $3 b$ ). Coexpression of hCLSP- $\triangle \mathrm{C} 1$, hCLSP- $\triangle \mathrm{C} 2$ or hCLSP with V642I-APP rescued V642I-APP-induced death (Figure 3c), whereas coexpression of hCLSP- $\Delta \mathrm{N} 1$ did not (Figure $3 e$, hCLSP$\Delta \mathrm{N} 1)$. Immunoblot analysis showed that hCLSP- $\Delta \mathrm{C} 1$, hCLSP- $\triangle \mathrm{C} 2$ and hCLSP were secreted into the $\mathrm{CM}$ (Figure 3d, left bottom), whereas hCLSP- $\Delta$ N1 was not (Figure $3 f$, left bottom). To investigate whether the inability of hCLSP- $\Delta \mathrm{N} 1$ to inhibit V642l-APP-induced cell death is because the mutant is not secreted or is not protective, we constructed the vector encoding ss-hCLSP- $\Delta N 1$. This is hCLSP- $\Delta \mathrm{N} 1$ with a 25 amino-acid sequence containing the mouse insulin-like growth factor-1 signal sequence (corresponding to amino acids Ser12-Gly33 of mouse insulin-like growth factor-1) attached to the N-terminus of hCLSP- $\Delta N 1$ to drive secretion. The ss-hCLSP- $\Delta \mathrm{N} 1$ was secreted into the CM (Figure 3f, left bottom) and inhibited V642I-APP-induced death (Figure $3 e$ ). These results indicate that the secretion of hCLSP is essential for its activity and that the $\mathrm{N}$-terminal 39-amino-acid region of hCLSP is essential for the secretion of hCLSP but are dispensable for its protective activity.

Similar to ss-hCLSP- $\Delta \mathrm{N} 1$, a vector encoding ss-hCLSP$\triangle \mathrm{N} 2$, a deletion mutant of hCLSP, in which an $\mathrm{N}$-terminal region (a.a. 2-60) containing the Humanin-like domain were deleted (Figure $3 b$ ) and the amino acid sequence Ser12Gly33 of mouse insulin-like growth factor-1 was attached to at its N-terminus, was constructed. Coexpression of ss-hCLSP$\triangle \mathrm{N} 2$ did not inhibit V642I-APP-induced death (Figure $3 \mathrm{~g}$ ). Although the amount of secreted ss-hCLSP- $\Delta \mathrm{N} 2$ was smaller than ss-hCLSP- $\Delta \mathrm{N} 1$ (Figure $3 \mathrm{~h}$ ), considering the sensitivity of the Myc antibody in the immunoblot analysis, we roughly estimated that the concentration of secreted ss-hCLSP- $\Delta \mathrm{N} 2$ in the $\mathrm{CM}$ was more than $10 \mu \mathrm{M}$ (Figure 3h). These results together suggest that amino acids 40-61 of hCLSP (TGKNLSEAGLRKLISEVDSDGD), which is named here the endogenous Humanin-like region (EHR)) and contains the Humanin-like domain (Figures $1 \mathrm{c}$ and $3 \mathrm{~b}$ ), is the core region responsible for the CLSP activity.

A synthetic EHR peptide exerts Humanin-like activity. Next, we examined the ability of a chemically synthesized EHR peptide to rescue V6421-APP-induced cell death. Treatment with Humanin or EHR at a concentration of $10 \mu \mathrm{m}$, but not $10 \mathrm{~nm}$, inhibited V642I-APP-induced death (Figures $4 \mathrm{a}$ and $\mathrm{b}$ ). This result confirms that EHR is the core region responsible for the activity of hCLSP and indirectly support the hypothesis that secreted hCLSP prevents the V642I-APP-induced death by acting outside the cells.

The S14G mutation of Humanin (HNG) increases the Humanin activity by 1000 times. ${ }^{1,2,41}$ Therefore, we simultaneously examined the activity of S54G-EHR (termed EHR-G), as Ser54 of hCLSP is analogous to Ser14 of Humanin. However, the S54G substitution in the EHR peptide did not apparently appear to potentiate its activity (Figure 4a).

hCLSP is more potent than Humanin. For the preparation of recombinant hCLSP, we affinity-purified glutathione-Stransferase (GST)-hCLSP-MycHis (hCLSP N-terminally tagged with GST and C-terminally tagged with MycHis) from bacteria expressing GST-hCLSP-MycHis using glutathione beads, released hCLSP-MycHis from GST bound to the glutathione beads with thrombin and determined the 
a

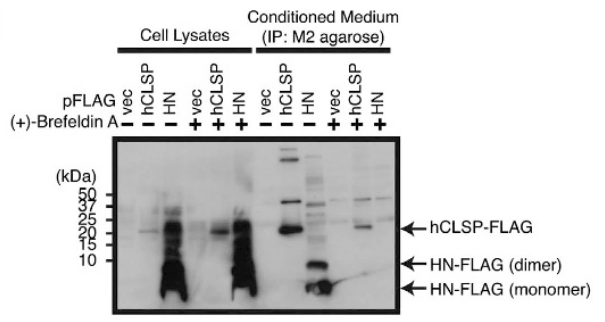

b

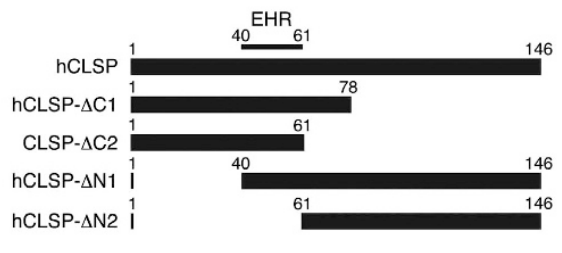

f
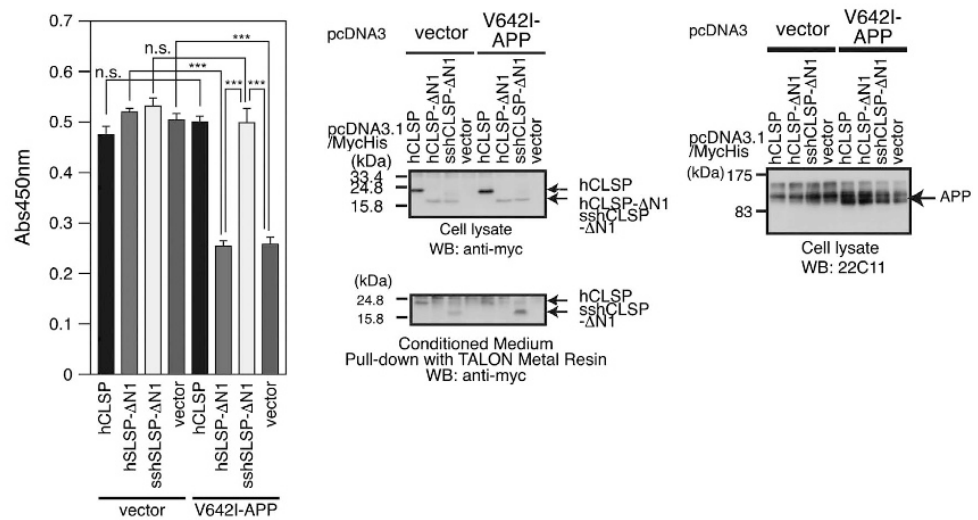

C

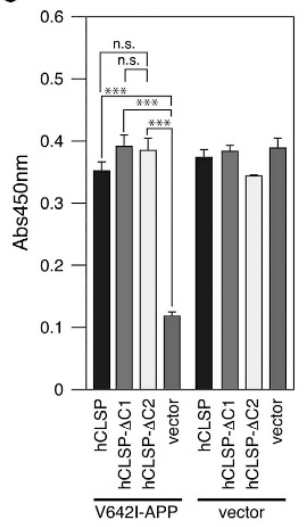

e

d

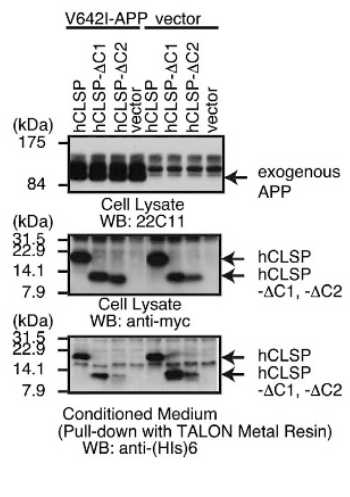

g

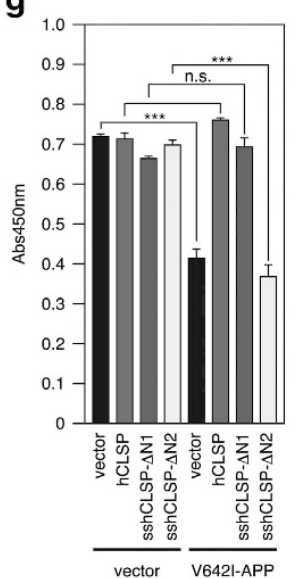

h

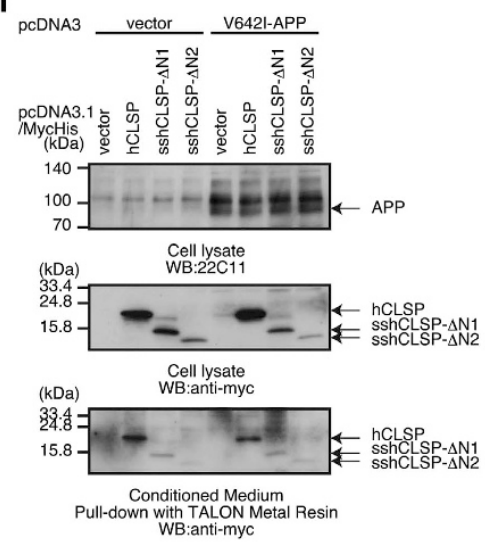

Figure 3 Secretion is essential for hCLSP activity. (a) SH-SY5Y cells were transfected with the empty pFLAG vector (vec) or the pFLAG vector encoding hCLSP or Humanin (HN) (C-terminally FLAG-tagged), and coincubated with or without (+)-Brefeldin A (BFA) $(20 \mathrm{ng} / \mathrm{ml})$ for $6 \mathrm{~h}$. The CM were subjected to the immunoprecipitatation with M2 FLAG-antibody-conjugated agarose. The resulting immunoprecipitates and input cell lysates were subjected to SDS-PAGE and immunoblot analysis with the M2 FLAG antibody. (b) The structures of the hCLSP deletion mutants (all C-terminally tagged with Mychis). EHR; endogenous Humanin-like region (see text). (c-h) SH-SY5Y cells, co-transfected with the empty pcDNA3 vector (vector) or pcDNA3-V642I-APP (V642I-APP) together with the pcDNA3.1/MycHis vector (vector) or the pcDNA3.1/MycHis encoding hCLSP or one of hCLSP deletion mutants (all C-terminally tagged with MycHis), were harvested for WST-8 assays (c, e and $\mathbf{g}$ ). The cell lysates were subjected to SDS-PAGE and immunoblot analysis with indicated antibodies $(\mathbf{d}, \mathbf{f}$ and $\mathbf{h})$. CM were also pulled down with Talon metal beads and the precipitates were subjected to SDS-PAGE and immunoblot analysis with indicated antibodies (d, bottom panel; $\mathbf{f}$, left bottom panel; $\mathbf{h}$, bottom panel). The $6 \times$ His antibody was used only for the detection of hCLSP, hCLSP- $\Delta \mathrm{C} 1$ and hCLSP- $\Delta \mathrm{C} 2$ in the CM in (d) ${ }^{* * *} P<0.001$; n.s., not significant in (c), (e), and (g)

approximate concentration of hCLSP-MycHis in the supernatant (Supplementary Figure S4A). In parallel, we affinitypurified hCLSP-MycHis from the CM of COS7 cells that had been transfected with a hCLSP-MycHis-expressing vector (Figure 5a, hCLSP) or the backbone vector as a control (Figure 5a, NC). Because the samples contained considerable amount of contaminants, the concentration of hCLSPMycHis, produced in COS7 cells, was estimated by the comparison to known amounts of hCLSP-MycHis, purified from bacteria, detected by SDS-PAGE analysis and hypersensitive Coomassie blue staining (Supplementary Figure S4B). hCLSP-MycHis, affinity-purified from the CM of COS7 cells, inhibited neuronal death in a dose-responsive manner (Figures $5 \mathrm{~b}$ and $\mathrm{c}$ ). We estimated that the $\mathrm{EC}_{50}$ of the hCLSP protective activity was $10-100 \mathrm{pm}$. This result indicates that hCLSP is $10^{4}-10^{5}$ times more potent than Humanin $\left(E C_{50}=1-10 \mu \mathrm{m}\right)$. As the EHR peptide had less activity than hCLSP-MycHis (Figure 4b), regions other than the EHR of hCLSP or other proteins that form a complex with hCLSP might increase the Humanin-like activity of the EHR. 
a

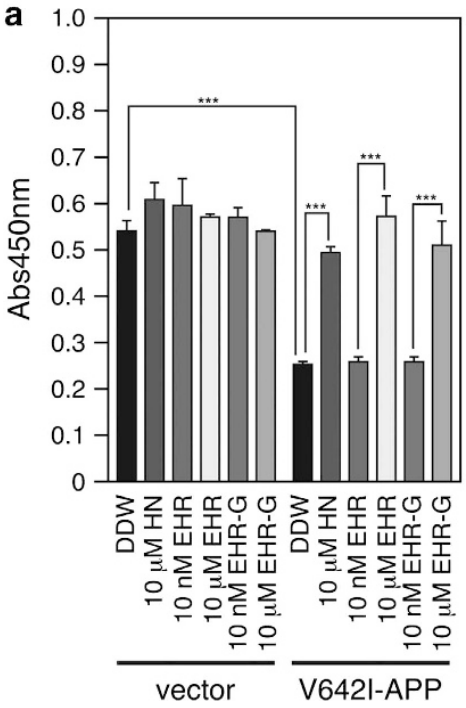

b

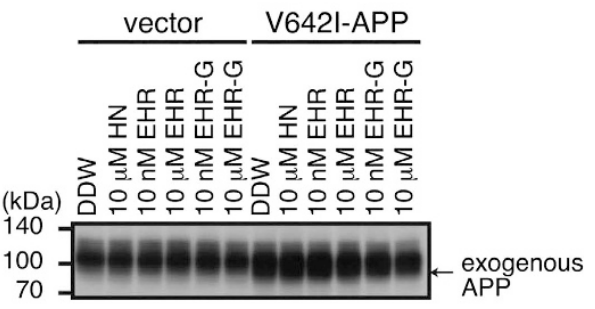

Figure 4 The EHR of hCLSP inhibits V642I-APP-induced death. (a, b) SH-SY5Y cells, transfected with the empty pcDNA3 vector (vector) or pcDNA3-V642I-APP (V642I-APP) and coincubated with the indicated concentrations of synthetic EHR, EHR-G or Humanin (HN), were harvested for WST-8 assays (a). ${ }^{* \star} P<0.001$. The cell lysates were subjected to SDS-PAGE and immunoblot analysis with the APP antibody $22 \mathrm{C} 11$ (b)
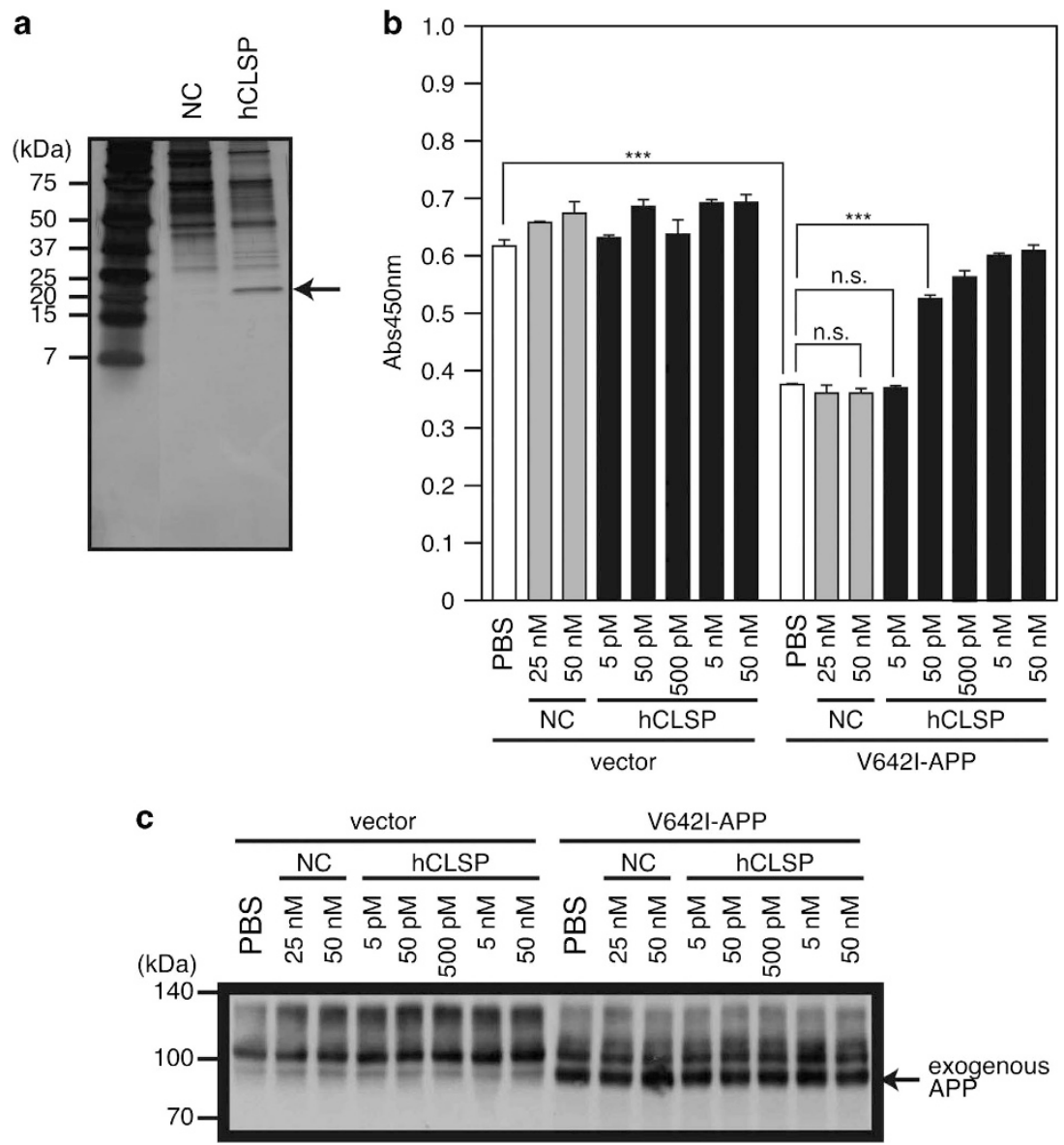

Figure 5 CLSP is more potent than Humanin. (a) SDS-PAGE analysis and silver staining of hCLSP-MycHis (hCLSP) and negative control (NC) that were partially purified from the CM of COS7 cells, transfected with pcDNA3.1/MycHis-hCLSP and the vector, respectively, with TALON Metal Resin. (b, c) SH-SY5Y cells, transfected the empty pcDNA3 vector (vector) or pcDNA3-V642I-APP (V642I-APP) and coincubated with the indicated concentrations of partially purified hCLSP-MycHis or NC, were harvested for WST-8 assays (b). ${ }^{* *} P<0.001$; n.S., not significant. The indicated concentrations of the NCs corresponded to the concentrations of compared purified hCLSP-MycHis. The cell lysates were subjected to SDS-PAGE and immunoblot analysis with the APP antibody (c) 
hCLSP inhibits neuronal death via the htHNR.

Previously, we showed that the binding of Humanin to the htHNR, led to an increase in the STAT3 phosphorylation proportionally to the activation of STAT3. ${ }^{30,31,42}$ Herein, coincubation of SH-SY5Y cells with recombinant hCLSPMychis (100 pm or $100 \mathrm{~nm}$ ), produced in COS7 cells, or synthetic Humanin $(10 \mu \mathrm{m})$ or S14G-Humanin (termed HNG; a Humanin derivative with a 1000-fold stronger activity) $(100 \mathrm{~nm})$, increased the level of the phosphorylation of STAT3 at Tyr705 (Figure 6a).

Humanin inhibits AD-related neuronal death by binding to the htHNR. ${ }^{30}$ Consistent with this, the hCLSP-induced inhibition of neuronal death was attenuated in the presence of a neutralizing antibody against gp130 (Figures 6b and c), CNTFR or WSX-1 (Supplementary Figures S5a-C and S6a-c). These results suggest that the CLSP-mediated inhibition of neuronal death, like that of HNG, occurs via htHNR.

The EHR of hCLSP binds to and oligomerizes the htHNR. Previously, we also showed that the association of the recombinant extracellular region of WSX-1 (WSX-1-ED), CNTFR-ED and gp130-ED was induced by the coincubation with Humanin in an in vitro htHNR reconstitution assay. ${ }^{30}$ Similarly, WSX-1-ED, CNTFR-ED and gp130-ED were incubated with $100 \mathrm{~nm}$ recombinant hCLSP-MycHis, produced in bacteria. Associations between WSX-1-ED and CNTFR-ED, between WSX-1-ED and gp130-ED and between WSX-1-ED and hCLSP, were detected (Figure 6d, HNR reconstitution assay, left panels, hCLSP). By contrast, no associations were detected in the absence of hCLSPMycHis (that is, in the GST control condition) (Figure $6 \mathrm{~d}$, HNR reconstitution assay, left panels, GST).

To determine the minimal region of hCLSP that binds to and oligomerizes the htHNR, we next assessed the oligomerization of htHNR in the presence of hCLSP deletion mutants (all C-terminally tagged with MycHis) (Supplementary Figure S7), produced in bacteria, using the same in vitro reconstitution assay. hCLSP, hCLSP- $\Delta$ C1, hCLSP- $\Delta N 1$ and EHR bound to and oligomerized the htHNR, whereas hCLSP- $\Delta \mathrm{N} 2$ did not (Figure 6d, HNR reconstitution assay). Ten micromolars of HNG, added in the assays, prevented EHR (100 nM) from binding to the htHNR and, in place of EHR, HNG bound to and oligomerized the htHNR (right panels), suggesting that HNG competed with EHR for binding to htHNR. These results together indicate that EHR is the core region of hCLSP that binds to and oligomerizes htHNR. We confirmed that the treatment with hCLSP, hCLSP- $\Delta$ C1, hCLSP- $\Delta \mathrm{N} 1$ or EHR increased the STAT3 phosphorylation in the SH-SY5Y cells, whereas the treatment with GST or hCLSP- $\Delta \mathrm{N} 2$ did not (Supplementary Figure S8).

\footnotetext{
Mouse CLSP inhibits V642I-APP-induced death of F11 neurohybrid cells and primary cortical neurons. Two mouse counterparts for hCLSP, originally named Scarf and Scarf2, ${ }^{38}$ were renamed as mouse CLSP-1 (mCLSP-1) and mCLSP-2 in the current study. The amino acid similarity between hCLSP and mCLSP-1 or mCLSP-2 is $\sim 60 \%$. ${ }^{38} \mathrm{We}$ confirmed the previous finding that mCLSPs were highly expressed in the skin (Supplementary Figure S9, skin), ${ }^{38}$ and found that this expression levels did not differ between young
}

and aged mice (Supplementary Figure S9, skin). The levels of mCLSP-1 and mCLSP-2 expression were markedly lower in the brain than in the skin (Supplementary Figure S9, hippocampus).

Next, we tested whether mCLSP-1 and mCLSP-2 have neuroprotective activities. Coexpression of $\mathrm{mCLSP}-1$ or mCLSP-2 with V642I-APP inhibited the V642I-APP-induced death of $\mathrm{F} 11$ neurohybrid cells (Figures $7 \mathrm{a}-\mathrm{C}$ ). ${ }^{1,2}$ The same experiment was performed using mouse primary cortical neurons, a more physiologically relevant model; expression of mCLSP-1 reduced V642I-APP-induced neuronal apoptosis (Supplementary Figure S10).

CLSP circulates in the blood and is transported across the blood-CSF barrier into the CSF. Although CLSP appears to function mainly in the CNS, the expression of CLSP is assumed to be very low in the CNS and high in the skin tissues (Supplementary Figure S9). ${ }^{37,38}$ These results led us to investigate whether CLSP could be transported via the blood circulation across the blood-CSF barrier (or the blood-brain barrier) into the CNS in the mouse. At $1 \mathrm{~h}$ after an i.p. injection of $5 \mathrm{nmol}$ of hCLSP-MycHis, produced in bacteria (Supplementary Figure S4), the concentration of hCLSP-MycHis reached $\sim 5 \mathrm{~nm}$ in the CSF (Figure $8 \mathrm{a}$, top) and $\sim 500 \mathrm{~nm}$ in the blood serum (Figure $8 \mathrm{a}$, bottom), whereas hCLSP-MycHis was not detected when intranasally (i.n.) administered (Figure 8a, top). Using another mouse, we confirmed that the i.p. administration of hCLSP-MycHis caused an appearance of hCLSP-MycHis at approximately similar concentrations in both serum and CSF (Figure 8b). Importantly, immunoblot analysis with anti-mouse IgG antibody showed that the amount of IgG heavy chain in the $0.1 \mu \mathrm{l}$ sera was much higher than that in $10 \mu \mathrm{l} \mathrm{CSF}$, indicating that the appearance of hCLSP-MycHis in the CSF was not caused by contamination of blood during aspiration of CSF from mice. This result suggests that hCLSP is transported via the blood circulation across the blood-CSF barrier (and also the blood-brain barrier) to the CSF (and the CNS). Note that the hCLSP-MycHis concentration in the CSF was $\sim 1 / 100$ of that in the serum at $1 \mathrm{~h}$ after the i.p. injection of hCLSPMycHis.

Next, we attempted to immunoprecipitate hCLSP from $100 \mu \mathrm{l}$ of blood plasma that was collected from three healthy male volunteers. A $15 \mathrm{kDa}$ protein, which was recognized by the hCLSP antibody in the immunoblot analysis, existed in the immunoprecipitates with the hCLSP antibody but not in the immunoprecipitates with the preimmune serum (Figure $8 \mathrm{c}$ ). The size of the immunoprecipitated $15 \mathrm{kDa}$ protein was a little smaller than that of the recombinant hCLSP-mycHis in the CM of COS7 cells (Figure 8c). This result suggests that the protein in the human blood plasma may be the endogenous hCLSP. If the immunoprecipitation efficiency were $100 \%$, the concentration of hCLSP would be estimated to be $\sim 5 \mathrm{~nm}$ in normal human blood plasma.

\section{Discussion}

It is possible that the CLSP detected in the CM was not secreted but was derived from cells that had died during the transfection procedure. However, we concluded that it is 
a

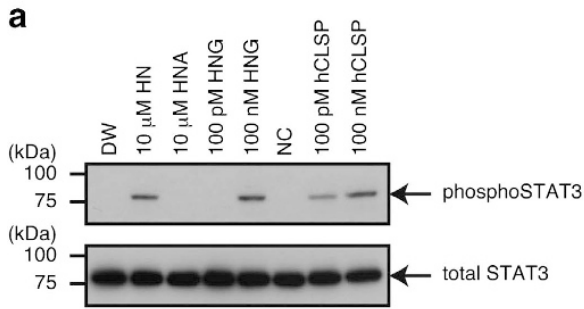

b

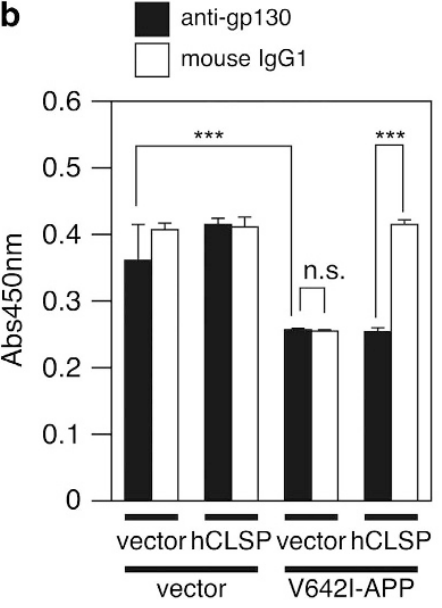

C

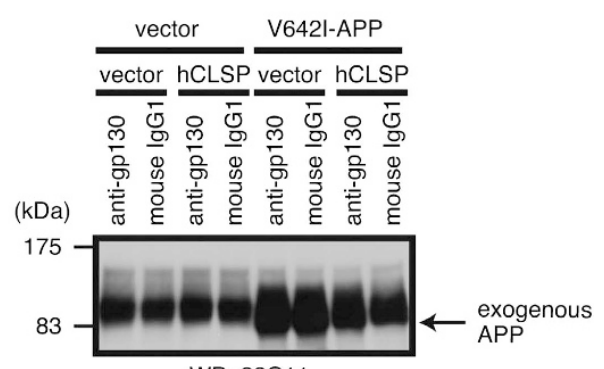

WB: 22C11
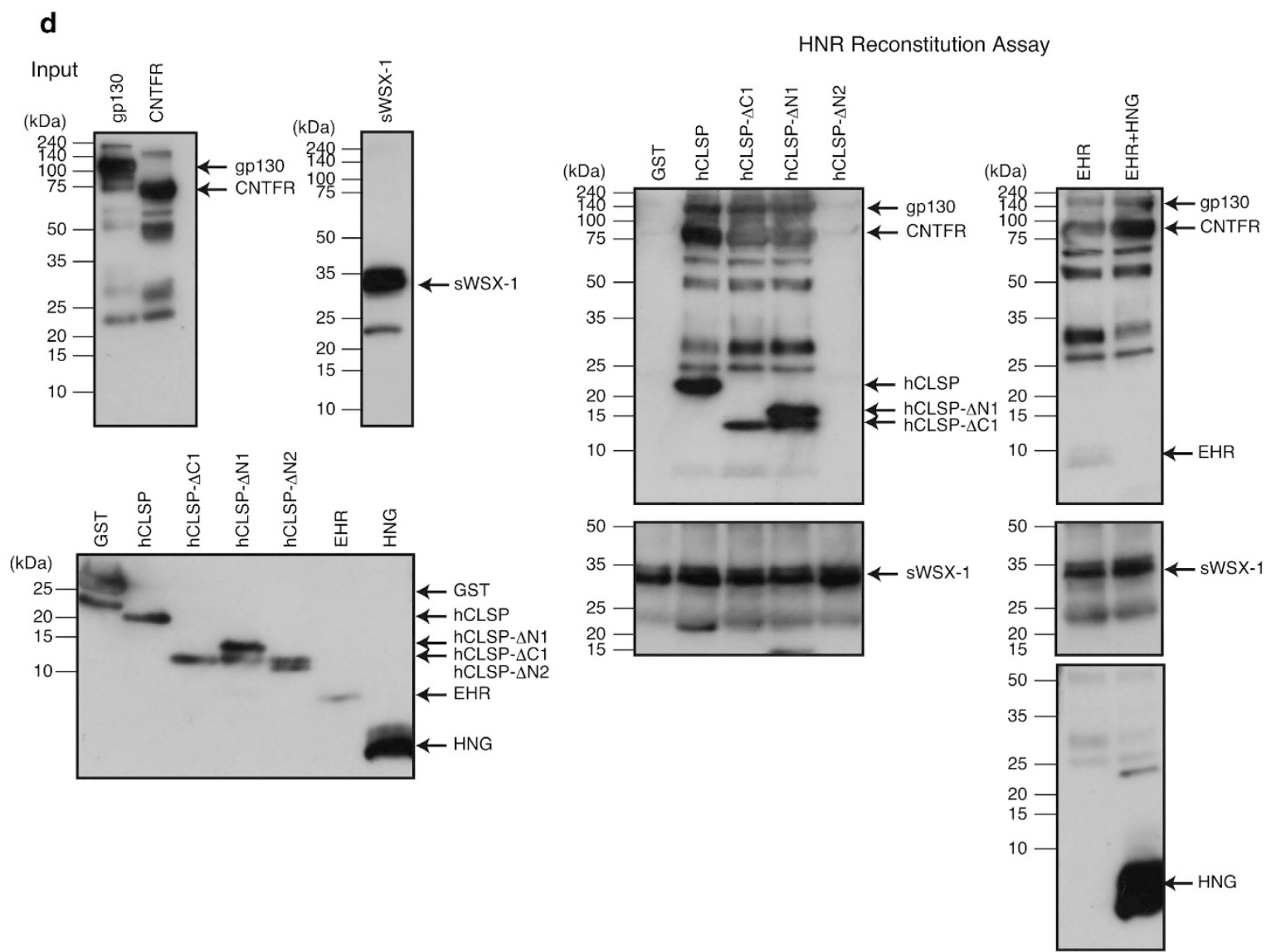

Figure 6 hCLSP exerts a protective activity by binding to the heterotrimeric Humanin receptor. (a) Serum-starved SH-SY5Y cells were incubated with indicated concentrations of Humanin (HN), S14G-HN (HNG; a Humanin derivative with a 1000-fold stronger activity), C8A-HN (HNA; a HN derivative with null activity) or recombinant hCLSP-MycHis (hCLSP), purified from the CM of COS7 cells, and then harvested for SDS-PAGE and immunoblot analysis with the phosphoSTAT3 (Tyr705) and total STAT3 antibodies. (b, c) SH-SY5Y cells, co-transfected with the empty pcDNA3 vector (vector) or pcDNA3-V642I-APP (V642I-APP) together with the pcDNA3.1/MycHis vector (vector) or pcDNA3.1/MycHis-hCLSP (hCLSP) and coincubated with neutralizing antibody to gp130 or control lgG ( $1 \mu \mathrm{g} / \mathrm{ml})$, were harvested for WST-8 assays (b). ${ }^{* * *} P<0.001$; n.S., not significant. The cell lysates were subjected to SDS-PAGE and immunoblot analysis with the APP antibody (c). (d) CNTFR-extracellular domain (ED)-Fc-6 $\times$ His (indicated CNTFR) and gp130-ED-Fc-6 $\times$ His (gp130) were coincubated with WSX-1-ED-FLAG (WSX-1), which was beforehand immobilized onto the M2 FLAG-antibody-conjugated agarose, in the presence of $100 \mathrm{~nm}$ of $h$ CLSP-MycHis (hCLSP) or one of its deletion mutants ( $h C L S P-\Delta C 1, h C L S P-\Delta N 1, h C L S P-\Delta N 2, E H R$ ) (all C-terminally tagged with MycHis) or GST-MycHis (GST), produced in bacteria, as a negative control (Supplementary Figure S7). In the HNG competition assay, $10 \mu \mathrm{m}$ of HNG was added (indicated EHR $+\mathrm{HNG}$ ). After the agaroses were washed, the resulting immunoprecipitates (HNR reconstitution assay), attached to the agaroses, and input recombinant proteins (Input), were subjected to SDS-PAGE and immunoblot analysis with anti-6 $\times$ His antibody (used for the detection of CNTFR-ED, gp130-ED, hCLSP, hCLSP deletion mutants and GST), the FLAG antibody (used for the detection of WSX-1-ED) or PO4 (used for the detection of HNG) 
a

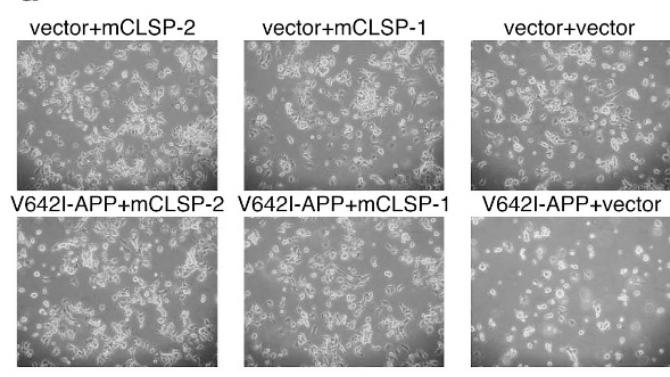

b

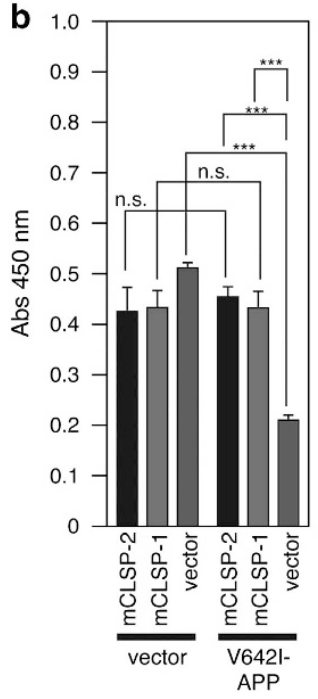

C

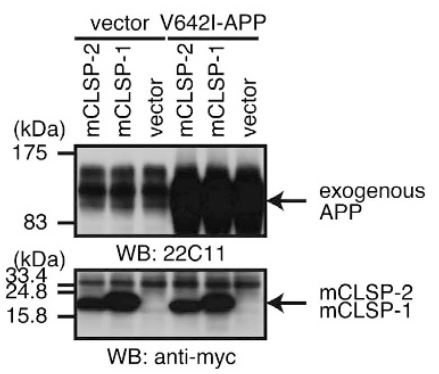

Figure 7 Co-expression of mCLSP-1 or mCLSP-2 inhibits V642I-APP-induced murine neuronal death. (a, b) F11 cells, were co-transfected with the empty pcDNA3 vector (vector) or pcDNA3-V642I-APP (V642I-APP) together with the pcDNA3.1/MycHis vector (vector), pcDNA3.1/MycHis-mCLSP-1 (mCLSP-1) or pcDNA3.1/MycHis-mCLSP-2 (mCLSP-2). At $72 \mathrm{~h}$, the cells were harvested for microscopic analysis (a) and WST-8 assays (b). ${ }^{* \star \star} P<0.001 ; \mathrm{n} . \mathrm{S}$., not significant. The cell lysates were subjected to SDSPAGE and immunoblot analysis with indicated antibodies (c)

highly likely that CLSP was secreted via a physiological secretion process, because (i) the treatment with BFA diminished the CLSP secretion (Figure 3a), (ii) the secretion was essential for the CLSP-induced inhibition of the ADrelevant death (Figures $3 e$ and f) and (iii) a considerable amount of CLSP appeared to circulate in the blood in vivo (Figures $8 a$ and $b$ ).

CLSP lacks the typical secretion signal sequence (Figure 1), although the treatment of cells with BFA diminished the secretion of CLSP (Figure 3a). CLSP binds to several intracellular proteins, including nucleolin, ERp72, 14-3-3 $\beta$ and 14-3-3 $\sigma^{43}$ It is possible that CLSP forms a complex with an unidentified protein $\mathrm{X}$ that has the typical signal sequence for secretion, thereby enabling CLSP to be secreted via the conventional secretory pathway in complex with this protein (that is, CLSP/X complex). ${ }^{41}$

Mutation of the Pro3, Cys8, Leu9, Leu12, Thr13, Ser14 or Pro19 of the Humanin's core region (Figure 1c) to alanine nullifies the Humanin activity, whereas that of the other amino acid does not. ${ }^{2,8}$ Among these functionally important amino acids, Leu9, Leu12 and Ser14 are conserved in the Humaninlike domain of hCLSP, whereas Pro3, Cys8, Thr13 and Pro19 are changed to asparagine, glutamine, isoleucine and aspartate, respectively. The EHR of hCLSP had the Humanin-like activity (Figure 4) and bound to and oligomerized the htHNR (Figure 6d). Therefore, it is highly likely that the replacement of Pro3, Cys8, Thr13 or Pro19 by asparagine, glutamine, isoleucine and aspartate, respectively, does not impair Humanin activity.

The expression of CLSP was previously shown to be limited to the skin tissues. ${ }^{37,38,44}$ In agreement, the immunohistochemical analysis showed that CLSP was not apparently expressed in the hippocampus and other brain regions (Supplementary Figure S9 and other negative data not shown). However, inconsistent with this finding, the immunoprecipitation experiment showed that CLSP was expressed in cultured neuronal NT2 cells and glial SF268 cells (Figure 1a). It is possible that the sensitivity of the CLSP antibodies used was not sufficient to detect low levels of endogenous CLSP in the brain; therefore, one should be cautious in concluding that CLSP is not expressed in the CNS.

I.p. injected recombinant hCLSP-MycHis was transported across the blood-CSF barrier or the blood-brain barrier into the CSF in mice (Figures $8 a$ and $b$ ). The CSF concentration of hCLSP-MycHis was $1 / 100$ of the serum concentration of hCLSP-MycHis at $1 \mathrm{~h}$ after the i.p. administration in mice (Figures $8 \mathrm{a}$ and $\mathrm{b}$ ). The plasma concentration of hCLSP was estimated to be $\sim 5 \mathrm{~nm}$ in healthy middle-aged persons (Figure $8 \mathrm{c}$ ) and the $\mathrm{EC}_{50}$ of hCLSP was $10-100 \mathrm{pm}$ (Figure $5 \mathrm{~b}$ ). If the majority of hCLSP in the human CNS is transported from the skin via the blood circulation across the blood-CSF or blood-brain barrier in a fashion similar to mice, we could estimate that endogenous hCLSP in the human CNS may reach a concentration sufficient for its protective activity. Unfortunately, however, the hCLSP detection methods that are currently available have been unable to detect hCLSP at the concentrations below $100 \mathrm{pm}$.

Some bioactive peptides, secreted from the peripheral tissues, are transported via the blood circulation into the CNS where they exhibit physiological activities. For instance, ghrelin and leptin, secreted from the endocrine cells of the stomach and the adipose tissues, respectively, are transported across the blood-brain barrier, and affect food intake via the hypothalamus. ${ }^{45}$ They may also affect neurons in the memory regions of the brain including the hippocampus. ${ }^{45}$ The current study presents another example. Importantly, CLSP may be the first bioactive peptide that is mainly secreted by the skin and exerts its major activity in the CNS.

In conclusion, we have shown that CLSP is a potent agonist for the htHNR and circulates in human blood at the concentration sufficient for its activity (Figure 8 ). This finding 
a
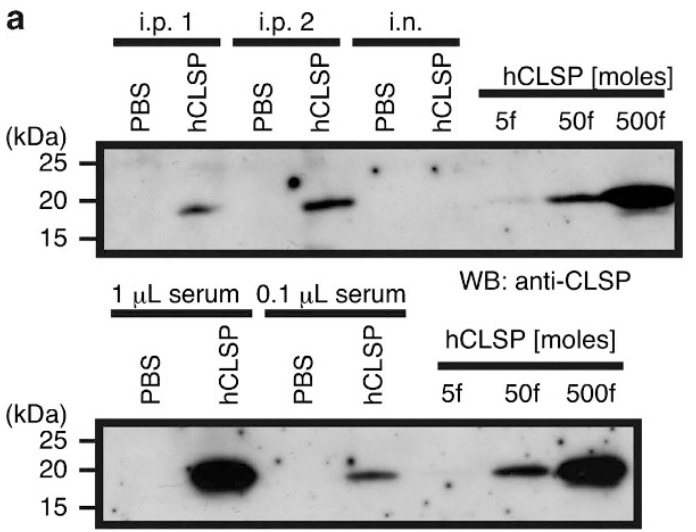

WB: anti-CLSP b $\quad 0.1 \mu \mathrm{L} \quad 10 \mu \mathrm{L}$
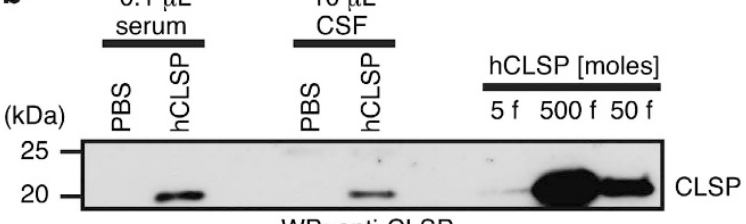

WB: anti-CLSP

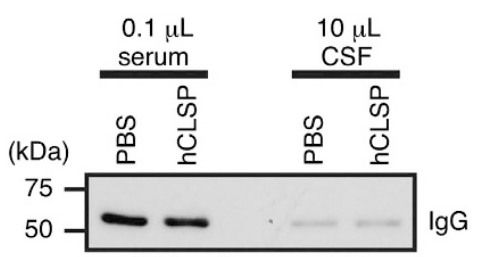

WB: anti-mouse $\lg G$
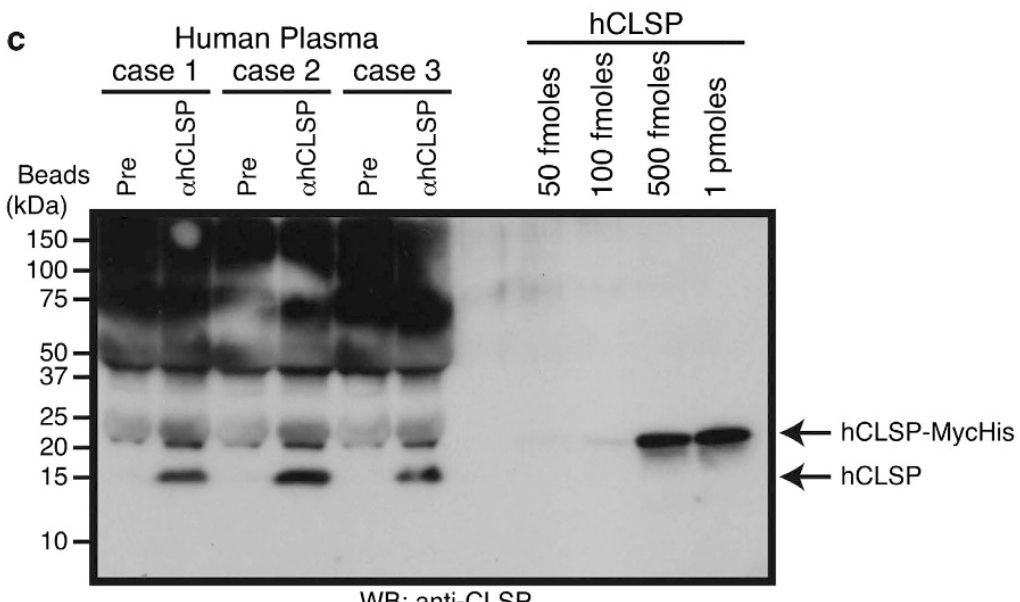

WB: anti-CLSP

Figure 8 CLSP is transported across the blood-CSF barrier into the CSF. (a) Purified hCLSP-MycHis, which was generated in E. coli, was i.p. injected into mice. For i.p.1, $100 \mu \mathrm{l}$ of the sample containing $1.7 \mathrm{nmol}$ of $\mathrm{hCLSP}$, was injected into a mouse three times with an interval of $1 \mathrm{~h}$. For i.p.2, $300 \mu \mathrm{l}$ of the sample containing $5.1 \mathrm{nmol}$ of hCLSP was injected at once. For i.n. administration, $0.34 \mathrm{nmol}$ of $\mathrm{hCLSP}-\mathrm{MycH}$ is in $20 \mu \mathrm{l}$ of PBS was i.n. administered at once. At $1 \mathrm{~h}$ from the last administration of $\mathrm{hCLSP-MycHis,}$ CSFs were collected from the i.p.1, i.p.2 and i.n. mice and $10 \mu \mathrm{l}$ of CSF was analyzed by SDS-PAGE and immunoblot analysis using the antibody against hCLSP (top). The indicated amounts of serum, collected from i.p.2 mouse, were similarly analyzed (bottom). Indicated femtomolar amounts of recombinant hCLSP were similarly processed for the estimation of the hCLSP amounts. The possible contamination of blood to CSF during the collection of CSF was estimated to be far less than $1 \%$. (b) An experiment similar to that in the panel (a) was performed using another mouse. At $1 \mathrm{~h}$ affter i.p. injection of $5.0 \mathrm{nmol}$ of hCLSP-MycHis, serum and CSF were collected. $0.1 \mu$ l of serum and $10 \mu \mathrm{l}$ of CSF were analyzed with SDS-PAGE and immunoblot analysis with antibodies to hCLSP (top) and mouse lgG (bottom). (c) Blood plasma samples (100 $\mu$ l) from three normal middle-aged males were subjected to the immunoprecipitation with CLSP antibody (anti-hCLSP)- or preimmune (Pre) serum-conjugated protein G-sepharose 4B. The immunoprecipitates were then fractionated by SDS-PAGE and immunobloted with CLSP antibody. Indicated amounts of recombinant hCLSP-MycHis, produced in bacteria, was simultaneously analyzed for the estimation of the hCLSP amounts in the plasma

greatly contributes to the establishment of the concept that the htHNR-mediated signal serves as a physiological signal.

\section{Materials and Methods}

Protein analysis and the identification of proteins by mass spectrometry. For analysis of cell lysates, cells were washed twice with phosphate-buffered saline (PBS), and suspended in $50 \mathrm{~mm}$ HEPES (pH7.4), $150 \mathrm{~mm} \mathrm{NaCl}, 0.1 \% \mathrm{NP}-40$ and protease inhibitor cocktail Complete (Roche Diagnostics, Almeda, CA, USA) (Buffer1). After freezing and thawing twice, the cell lysate was centrifuged at 15000 r.p.m. for $10 \mathrm{~min}$ at $4{ }^{\circ} \mathrm{C}$ and the supernatant was submitted to immunoprecipitation and/or analysis with Tris-Tricine SDS-PAGE and/or immunoblot analysis. For immunoprecipitation, supernatants were mixed with an antibody at $4{ }^{\circ} \mathrm{C}$ for $2 \mathrm{~h}$ and then mixed with $1: 1$ slurry of protein G-sepharose $4 \mathrm{~B}$ and rotated overnight before washing with Buffer1. In some experiments, proteins, fractionated by SDS-PAGE, were stained with silver staining (Wako Pure Chemicals, Tokyo, Japan) or hypersensitive Coomassie blue staining using GelCode Blue Stain Reagent (Thermo Scientific, Yokohama, Japan). Some protein bands were excised, digested with lysyl endopeptidase and analyzed by mass spectrometry.
Molecular mass analyses were performed by MALDI-TOF mass spectrometry (MS) using an ABI PerSeptiveBiosystem Voyager DE/RP (Applied Biosystems, Foster City, CA, USA). Identification of the proteins was conducted by comparing the molecular mass determined by MALDI-TOF/MS with theoretical peptide masses from the proteins registered in NCBInr database.

Recombinant CLSP and CLSP deletion mutants. GSThCLSP-MycHis and GST-hCLSP deletion mutants, C-terminally tagged with Mychlis, were expressed in $E$. coli BL-21 at $37^{\circ} \mathrm{C}$ for $4 \mathrm{~h}$ in $1 \mathrm{~mm}$ isopropyl-thio- $\beta$-Dgalactopyranoside. Purified GST-hCLSP-MycHis, bound to glutathione sepharose, was coincubated in PBS containing thrombin (1 unit/ml, GE Healthcare, Uppsala, Sweden, USA) at $25^{\circ} \mathrm{C}$ overnight. The thrombin target site was located between GST and hCLSP. The concentrations of hCLSP-MycHis and hCLSP deletion mutants, released from GST that bound to the glutathione sepharose, were determined using the BCA assay kit (Pierce, Rockford, IL, USA). For the purification of hCLSP-MycHis and mCLSP-1-MycHis from the CM of COS7 cells, the cells were transfected with pcDNA3.1/MycHis-hCLSP and cultured in DMEM containing 10\% FBS for $48 \mathrm{~h}$ before the collection of CM. Secreted CLSP-MycHis was affinity-purified using TALON Metal Resin (Clontech, Palo Alto, CA, USA) according to the manufacturer's instructions. Elutes were desalted with the Zeba Spin Column (Pierce). One-tenth volume of $10 \times$ 
PBS and an appropriate amount of protease cocktail complete solution (Roche Diagnostics) were added to the elutes. The concentration of the partially purified recombinant protein was estimated by its comparison with pure hCLSP-MycHis, which was produced in bacteria, using SDS-PAGE and hypersensitive Coomasie blue staining with GelCode Blue Stain Reagent (Thermo Scientific). Recombinant human gp130-ED-Fc- 6xHis was purchased from R\&D Systems (Minneapolis, MN, USA).

Antibodies. The polyclonal antibody PO4 was raised against the whole Humanin peptide in a rabbit. ${ }^{35}$ Ready-made antibodies were purchased from following companies: Myc from Invitrogen (Carlsbad, CA, USA; FLAG (M2) from Sigma-Aldrich (St Louis, MO, USA); APP (22C11 from Chemicon (Temecula, CA, USA); phoshoSTAT3 (Tyr705) from Cell Signaling Technology (Beverly, MA, USA); and STAT3 (C-20) and $6 \times$ His (His probe) from Santa Cruz Biotechnology (Santa Cruz, CA, USA). A rabbit polyclonal antibody against GST-hCLSP-MycHis was generated by immunization with recombinant GST-hCLSP-MycHis, which was produced in bacteria. Neutralizing antibodies to human gp130, CNTFR and WSX-1 were previously described. ${ }^{30}$

Genes, synthetic peptides and other materials. A hCLSP CDNA (GenBank: BC039172.1) was obtained from the I.M.A.G.E. Consortium/LLNL (MGC: 20994 IMAGE:4753280). The cDNAs for mCLSPs were PCR-amplified from mRNA of mouse skin tissues. The CDNAs encoding hCLSP, hCLSP deletion mutants, mCLSP-1 and mCLSP-2 were introduced into pcDNA3.1-MycHis, pFLAG and $\mathrm{pGEX}-2 \mathrm{~T}$ vectors to generate C-terminally MycHis-tagged, C-terminally FLAG-tagged and N-terminally GST-tagged proteins. The pGEX-2T vector encodes a site for the thrombin-mediated cleavage at the junction of GST and a transgene. The construct Ss-hCLSP- $\Delta \mathrm{N}$ encoded $h C L S P-\Delta N$ that is N-terminally fused to an artificial signal sequence, consisting of a Met-mouse insulin-like growth factor-1 (Ser12-Gly33)-Lys-Pro peptide. The pIRES-EGFP vector was purchased from Clontech. Humanin (HN), S14G-HN (HNG; a Humanin derivative with a 1000fold stronger activity), C8A-HN (HNA; a Humanin derivative with no activity), EHR and S54G-EHD (EHR-G) were synthesized by the Keck Foundation Biotechnology Resource Laboratory of Yale University.

Cells, cell death and cell viability. The mouse MLTL-1 Leydig cells, TM3 Leydig cells, human glioblastoma SF268 cells, were obtained from ATCC (Manassas, VA, USA). The human teratocarcinoma cell line NT2 were obtained from Stratagene (La Jolla, CA, USA).

Neuronal cell-death assays, related to $A D$, were first performed by Yamatsuji et al.. ${ }^{46}$ Human SH-SY5Y neuroblastoma cells, were grown in DMEM/Ham's F12 mixture (DMEM/F12) (Wako, Wakayama, Japan) containing 10\% FBS, seeded at $2 \times 10^{5} /$ well in six-well plates for $12-16 \mathrm{~h}$, transfected with the appropriate vectors for $3 \mathrm{~h}$ in the absence of serum and then cultured in DMEM/F12-10\% FBS and/or a rescue factor. At $24 \mathrm{~h}$ after the transfection, the media were replaced with DMEM/F12 containing N2 supplement (Invitrogen) and/or a rescue factor. At $48 \mathrm{~h}$ after the onset of the transfection, cells were harvested to perform cell viability assays using the WST-8 assay kit (Dojindo, Kumamoto, Japan) and their microscopic views were taken to show viable cells that were attached to cell plates (the conditions of photographing were not consistent among the figures). F11 neurohybrid cells are described in earlier studies. ${ }^{1,2} \mathrm{~F} 11$ cells, seeded at $7 \times 10^{4} /$ well in six-well plates in Ham's F12-18\% FBS for 12-16 h, were co-transfected with indicated vectors for $3 \mathrm{~h}$ in the absence of serum and were then incubated with Ham's F12-18\% FBS for $2 \mathrm{~h}$. At $5 \mathrm{~h}$ after the onset of the transfection, culture media were replaced by Ham's F12-10\% FBS. At $24 \mathrm{~h}$ after the transfection, the media were replaced by Ham's F12 containing N2 supplement. At $72 \mathrm{~h}$ after the onset of the transfection, the cells were harvested to perform cell viability assays. The cells were sometimes stained with calcein AM (Dojindo) at $48 \mathrm{~h}$ after the transfection.

CLSP-induced oligomerization of receptor subunits. This experiment was performed as previously described in detail. ${ }^{30}$ The extracellular domain (ED) of human WSX-1 (amino acids 1-390) was used in place of the ED of mouse WSX-1 in the current study. To generate recombinant human CNTFR-ED-Fc6xHis and human WSX-1-ED- FLAG, COS7 cells were transfected with a vector encoding each protein. At $48 \mathrm{~h}$ after the transfection, CM were harvested for precipitation with Ni NTA agarose (1:1 slurry; Invitrogen) or anti-FLAG antibody M2- conjugated agarose (1:1 slurry; Sigma-Aldrich). Bound CNTFR-ED-Fc-6xHis, washed with $10 \mathrm{~mm}$ imidazole solution, were eluted with $250 \mathrm{~mm}$ imidazole solution according to manufacturer's instructions. Eluted CNTFR-ED-Fc- 6xHis was desalted by Zeba Desalting Column (Pierce, Rockford, IL, USA) and then onetenth volume of $10 \times$ PBS was added to the desalted protein solution.
To induce the oligomerization, gp130-ED-Fc-6xHis $(1 \mu \mathrm{g})$ and CNTFR-ED- Fc$6 \mathrm{xHis}(1 \mu \mathrm{g})$ were coincubated with WSX-1-ED-FLAG (estimated to be $5-10 \mu \mathrm{g}$ ) immobilized onto anti-FLAG monoclonal antibody M2-conjugated agarose beads in $200 \mu$ l PBS containing 1\% Brij 96 (Sigma-Aldrich) in the presence of a peptide at $37^{\circ} \mathrm{C}$ for $6 \mathrm{~h}$. After being washed with the same buffer, oligomerized proteins bound to the beads were subjected to SDS-PAGE and immunoblot analysis.

Tyr705-phosphorylated STAT3. SH-SY5Y cells were cultured in DMEM/ F12 containing N2 supplement (Invitrogen) without FBS for $24 \mathrm{~h}$, treated with the appropriate factors for $30 \mathrm{~min}$ at $37^{\circ} \mathrm{C}$, and then harvested for immunoblot analysis. ${ }^{30}$

Experiments using animals and human samples. All of the experimental procedures were approved by the Institutional Human and Animal Experiment Committees of Tokyo Medical University.

Collection of serum and CSF from mice. At $1 \mathrm{~h}$ after the i.p. administration of hCLSP-MycHis (5.1 or $5.0 \mathrm{nmol}$ in PBS), produced in bacteria, into a mouse, blood and CSF were aspirated from the inferior vena cava and the brain after the mouse have been anesthetized with pentobarbital. CSF was not apparently contaminated by blood. The ratio of the possible contaminated blood volume to the volume of CSF was estimated to be far less than $1 \%$.

Immunoprecpitation of endogenous hCLSP from human blood plasma. For the preparation of high-quality antibody-conjugated beads, $200 \mu \mathrm{l}$ of serum and $800 \mu \mathrm{l}$ of Protein G Sepharose 4B (1:1 slurry, GE Healthcare) was coincubated at $4{ }^{\circ} \mathrm{C}$ overnight. After being washed with $0.2 \mathrm{M}$ sodium tetraborate (pH 9.0), the samples were suspended in $2 \mathrm{ml}$ of the same buffer, mixed with $200 \mu$ of $0.2 \mathrm{~m}$ dimethyl pimelimidate-2 $\mathrm{HCl}$, which is a cross-linker (Pierce) and incubated for $30 \mathrm{~min}$ at room temperature. After being washed, the beads were mixed with $10 \mathrm{ml}$ of $0.2 \mathrm{~m}$ ethanolamine and incubated for $2 \mathrm{~h}$ at room temperature for blocking. Human blood was aspirated from three middle-aged normal male volunteers. One hundred microliters of purified plasma was coincubated with $20 \mu$ of $1: 1$ slurry of GST-CLSP antibody or preimmune-conjugated beads at $4{ }^{\circ} \mathrm{C}$ overnight for immunoprecipitation.

Statistical analysis. All cell-death experiments were performed with $n=3$. All values in the figures are the mean \pm S.D. Statistical analysis was performed using one-way analysis of variance, followed by Bonferroni/Dunn post hoc analysis. All data were analyzed using StatView (version 5.0.1) software from SAS Institute (Cary, NC, USA) $\quad\left({ }^{\star \star \star} P<0.001 ; \quad{ }^{* \star} P<0.01 ; \quad{ }^{*} P<0.05\right.$; n.s., not significant).

\section{Conflict of Interest}

The authors declare no conflict of interest.

Acknowledgements. We are especially grateful to Ms. Takako Hiraki, Yuka Toyama and Tomoko Ohhara for essential assistance throughout the study. This study was in part supported by the National Institute of the Biomedical Innovation, the Japan Health Foundation, the Mitsui Life Social Welfare Foundation and the Daiwa Securities Health Foundation to MM. This work was also in part supported by the Grant-in-Aid for Scientific Research (B) (grant number 23390059 to MM) and (C) (grant number 225902417 to $\mathrm{YH}$ ). The authors are indebted to Dr. Clifford A Kolba (Ed.D., D.O., M.P.H.) and Associate Professor Edward F Barroga (D.V.M., Ph.D.) of the Department of International Medical Communications of Tokyo Medical University for their editorial review of the English manuscript.

\section{Author contributions}

$\mathrm{YH}$ and $\mathrm{MN}$ designed and performed the experiments and analyzed the data. MK and MT performed the experiments. Al performed the protein analysis by mass spectrometry. MM directed the study, came up with the hypothesis, designed the experiments, analyzed the data and wrote the manuscript.

1. Hashimoto $Y$, Niikura T, Tajima $H$, Yasukawa $T$, Sudo $H$, Ito $Y$ et al. A rescue factor abolishing neuronal cell death by a wide spectrum of familial Alzheimer's disease genes and A $\beta$. Proc Natl Acad Sci USA 2001; 98: 6336-6341. 
2. Hashimoto Y, Niikura T, Ito Y, Sudo H, Hata M, Arakawa E et al. Detailed characterization of neuroprotection by a rescue factor Humanin against various Alzheimer's diseaserelevant insults. J Neurosci 2001; 21: 9235-9245.

3. Wang D, Li H, Yuan H, Zheng M, Bai C, Chen L et al. Humanin delays apoptosis in K562 cells by downregulation of P38 MAP kinase. Apoptosis 2005; 10: 963-971.

4. Colon E, Strand ML, Carlsson-Skwirut C, Wahlgren A, Svechnikov KV, Cohen $P$ et al. Anti-apoptotic factor humanin is expressed in the testis and prevents cell-death in leydig cells during the first wave of spermatogenesis. J Cell Physiol 2006; 208: 373-385.

5. Kin T, Sugie K, Hirano M, Goto Y, Nishino I, Ueno S. Humanin expression in skeletal muscles of patients with chronic progressive external ophthalmoplegia. J Hum Genet 2006; 51: $555-558$.

6. Bachar AR, Scheffer L, Schroeder AS, Nakamura HK, Cobb LJ, Oh YK et al. Humanin is expressed in human vascular walls and has a cytoprotective effect against oxidized Idl-induced oxidative stress. Cardiovasc Res 2010; 88: 360-366.

7. Hoang PT, Park P, Cobb LJ, Paharkova-Vatchkova V, Hakimi M, Cohen P et al. The neurosurvival factor Humanin inhibits beta-cell apoptosis via signal transducer and activator of transcription 3 activation and delays and ameliorates diabetes in nonobese diabetic mice. Metabolism 2010; 59: 343-349.

8. Nishimoto I, Matsuoka M, Niikura T. Unraveling the role of Humanin. Trends Mol Med 2004; 10: 102-105.

9. Matsuoka M. Humanin; a defender against Alzheimer's disease? Recent Pat CNS Drug Discov 2009; 4: 37-44

10. Matsuoka M. Humanin signal for Alzheimer's disease. J Alzheim Dis 2011; 24: 27-32.

11. Xu X, Chua CC, Gao J, Hamdy RC, Chua BH. Humanin is a novel neuroprotective agent against stroke. Stroke 2006; 7: 2613-2619.

12. Xu X, Chua CC, Gao J, Chua KW, Wang H, Hamdy RC et al. Neuroprotective effect of humanin on cerebral ischemia/reperfusion injury is mediated by a PI3K/Akt pathway. Brain Res 2008; 1227: 12-18.

13. Lue Y, Swerdloff R, Liu Q, Mehta H, Hikim AS, Lee KW et al. Opposing roles of insulin-like growth factor binding protein 3 and humanin in the regulation of testicular germ cell apoptosis. Endocrinology 2010; 151: 350-357.

14. Muzumdar RH, Huffman DM, Calvert JW, Jha S, Weinberg $Y$, Cui $L$ et al. Acute humanin therapy attenuates myocardial ischemia and reperfusion injury in mice. Arterioscler Thromb Vasc Biol 2010; 30: 1940-1948.

15. Muzumdar RH, Huffman DM, Atzmon G, Buettner C, Cobb LJ, Fishman S et al. Humanin: a novel central regulator of peripheral insulin action. PLOS One 2009; 4: e6334.

16. Oh YK, Bachar AR, Zacharias DG, Kim SG, Wan J, Cobb LJ et al. Humanin preserves endothelial function and prevents atherosclerotic plaque progression in hypercholesterolemic ApoE deficient mice. Atherosclerosis 2010; 219: 65-73.

17. Mamiya T, Ukai M. [Gly(14)]-Humanin improved the learning and memory impairment induced by scopolamine in vivo. Br J Pharmacol 2001; 134: 1597-1599.

18. Krejcova G, Patocka J, Slaninova J. Effect of humanin analogues on experimentally induced impairment of spatial memory in rats. J Pept Sci 2004; 10: 636-639.

19. Kunesová G, Hlavácek J, Patocka J, Evangelou A, Zikos C, Benaki D et al. The multiple T-maze in vivo testing of the neuroprotective effect of humanin analogues. Peptides 2008; 29: 1982-1987.

20. Yamada M, Chiba T, Sasabe J, Nawa M, Tajima H, Niikura T et al. Implanted cannulamediated repetitive administration of A $\beta 25-35$ into the mouse cerebral ventricle effectively impairs spatial working memory. Behav Brain Res 2005; 164: 139-146.

21. Tajima H, Kawasumi M, Chiba T, Yamada M, Yamashita K, Nawa M et al. A Humanin derivative, S14G-HN, prevents amyloid- $\beta$-induced memory impairment in mice. J Neurosci Res 2005; 79: 714-723.

22. Chiba T, Yamada M, Hashimoto Y, Sato M, Sasabe J, Kita $Y$ et al. Development of femtomolar-acting humanin derivative named colivelin by attaching activity-dependen neurotrophic factor to its $\mathrm{N}$ terminus: characterization of Colivelin-mediated neuroprotection against Alzheimer's disease-relevant insults in vitro and in vivo. J Neurosci 2005; 25: 10252-10261.

23. Miao J, Zhang W, Yin R, Liu R, Su C, Lei G et al. S14G-Humanin ameliorates Aß25-35induced behavioral deficits by reducing neuroinflammatory responses and apoptosis in mice. Neuropeptides 2008; 42: 557-567.

24. Chiba T, Yamada M, Sasabe J, Terashita K, Shimoda M, Matsuoka M et al. Amyloid- $\beta$ causes memory impairment by disturbing the JAK2/STAT3 axis in hippocampal neurons. Mol Psychiatry 2009; 14: 206-222.

25. Niikura T, Sidahmed E, Hirata-Fukae C, Aisen PS, Matsuoka Y. A humanin derivative reduces amyloid Beta accumulation and ameliorates memory deficit in triple transgenic mice. PLoS One 2011; 6: e16259.
26. Zhang W, Zhang W, Li Z, Hao J, Zhang Z, Liu L et al. S14G-humanin improves cognitive deficits and reduces amyloid pathology in the middle-aged APPswe/PS1dE9 mice. Pharmacol Biochem Behav 2012; 100: 361-369.

27. Ashe KH, Zahs KR. Probing the biology of Alzheimer's disease in mice. Neuron 2010; 66 : $631-645$.

28. Guo B, Zhai D, Cabezas E, Welsh K, Nouraini S, Satterthwait AC et al. Humanin peptide suppresses apoptosis by interfering with Bax activation. Nature 2003; 423: 456-461.

29. Ying G, Iribarren P, Zhou Y, Gong W, Zhang N, Yu ZX et al. Humanin, a newly identified neuroprotective factor, uses the $G$ protein-coupled formylpeptide receptor-like-1 as a functional receptor. J Immunol 2004; 172: 7078-7085.

30. Hashimoto Y, Kurita M, Aiso S, Nishimoto I, Matsuoka M. Humanin inhibits neuronal cell death by interacting with a cytokine receptor complex or complexes involving CNTF receptor $\alpha /$ WSX-1/gp130. Mol Biol Cell 2009; 20: 2864-2873.

31. Hashimoto $\mathrm{Y}$, Kurita M, Matsuoka M. Identification of soluble WSX-1 not as a dominant-negative but as an alternative functional subunit of a receptor for an anti-Alzheimer's disease rescue factor Humanin. Biochem Biophys Res Commun 2009; 389: 95-99.

32. Ikonen M, Liu B, Hashimoto $\mathrm{Y}, \mathrm{Ma} \mathrm{L}$, Lee $\mathrm{K}$, Niikura $\mathrm{T}$ et al. Interaction between the Alzheimer's survival peptide humanin and insulin-like growth factor-binding protein 3 regulates cell survival and apoptosis. Proc Natl Acad Sci USA 2003: 100: 13042-13047.

33. Niikura $T$, Hashimoto $Y$, Tajima $H$, Ishizaka M, Yamagishi $Y$, Kawasumi $M$ et al. A tripartite motif protein TRIM11 binds and destabilizes Humanin, a neuroprotective peptide against Alzheimer's disease-relevant insults. Eur J Neurosci 2003; 17: 1150-1158.

34. Rossini L, Hashimoto Y, Suzuki H, Kurita M, Gianfriddo M, Scali C et al. VSTM2L is a novel secreted antagonist of the neuroprotective peptide Humanin. FASEB J 2011; 25: 1983-2000.

35. Tajima H, Niikura T, Hashimoto $\mathrm{Y}$, Ito $\mathrm{Y}$, Kita $\mathrm{Y}$, Terashita $\mathrm{K}$ et al. Evidence for in vivo production of Humanin peptide, a neuroprotective factor against Alzheimer's Diseaserelated insults. Neurosci Lett 2002; 324: 227-231.

36. Moretti E, Giannerini V, Rossini L, Matsuoka M, Trabalzini L, Collodel G. Immunolocalization of humanin in human sperm and testis. Fertil Steril 2010; 94: 2888-2890.

37. Méhul B, Bernard D, Simonetti L, Bernard MA, Schmidt R. Identification and cloning of a new calmodulin-like protein from human epidermis. J Biol Chem 2000; 275: 12841-12847.

38. Hwang M, Morasso Ml. The novel murine $\mathrm{Ca} 2+$-binding protein, Scarf, is differentially expressed during epidermal differentiation. J Biol Chem 2003; 278: 47827-47833.

39. Yamagishi Y, Hashimoto Y, Niikura T, Nishimoto I. Identification of essential amino acids in Humanin, a neuroprotective factor against Alzheimer's disease-relevant insults. Peptides 2003; 24: 585-595.

40. Nickel W, Rabouille C. Mechanisms of regulated unconventional protein secretion. Nat Rev Mol Cell Biol 2009; 10: 148-155.

41. Terashita K, Hashimoto Y, Niikura T, Tajima H, Yamagishi Y, Ishizaka M et al. Two serine residues distinctly regulate the rescue function of Humanin, an inhibiting factor of Alzheimer's disease-related neurotoxicity: functional potentiation by isomerization and dimerization. J Neurochem 2003; 85: 1521-1538.

42. Hashimoto $Y$, Suzuki H, Aiso S, Niikura T, Nishimoto I, Matsuoka M. Involvement of tyrosine kinases and STAT3 in Humanin-mediated neuroprotection. Life Sci 2005; 77: 3092-3104

43. Hwang J, Kalinin A, Hwang M, Anderson DE, Kim MJ, Stojadinovic O et al. Role of Scarf and its binding target proteins in epidermal calcium homeostasis. J Biol Chem 2007; 282: 18645-1853.

44. Hwang M, Kalinin A, Morasso MI. The temporal and spatial expression of the novel Ca++-binding proteins, Scarf and Scarf2, during development and epidermal differentiation. Gene Expr Patterns 2005; 5: 801-808.

45. Shan X, Yeo GSH. Central leptin and ghrelin signalling: Comparing and contrasting their mechanisms of action in the brain. Rev Endocr Metab Disord 2011; 12: 197-209.

46. Yamatsuji T, Matsui T, Okamoto T, Komatsuzaki K, Takeda S, Fukumoto H et al. G proteinmediated neuronal DNA fragmentation induced by familial Alzheimer's disease-associated mutants of APP. Science 1996; 272. 1349-1352.

(c) (i) $(9)$ Cell Death and Disease is an open-access journal published by Nature Publishing Group. This work is licensed under a Creative Commons Attribution-NonCommercialNoDerivs 3.0 Unported License. To view a copy of this license, visit http://creativecommons.org/licenses/by-nc-nd/3.0/

\section{Supplementary Information accompanies this paper on Cell Death and Disease website (http://www.nature.com/cddis)}

